\title{
Seasonal plasticity of auditory saccular sensitivity in "sneaker" type II male plainfin midshipman fish, Porichthys notatus
}

\author{
Ashwin A. Bhandiwad ${ }^{1}$ Elizabeth A. Whitchurch ${ }^{1}$ - Orphal Colleye Or, $^{1,}$ \\ David G. Zeddies ${ }^{3} \cdot$ Joseph A. Sisneros $^{1,4,5}$
}

Received: 14 September 2016 / Revised: 16 February 2017 / Accepted: 17 February 2017

(C) Springer-Verlag Berlin Heidelberg 2017

\begin{abstract}
Adult female and nesting (type I) male midshipman fish (Porichthys notatus) exhibit an adaptive form of auditory plasticity for the enhanced detection of social acoustic signals. Whether this adaptive plasticity also occurs in "sneaker" type II males is unknown. Here, we characterize auditory-evoked potentials recorded from hair cells in the saccule of reproductive and non-reproductive "sneaker" type II male midshipman to determine whether this sexual phenotype exhibits seasonal, reproductive statedependent changes in auditory sensitivity and frequency response to behaviorally relevant auditory stimuli. Saccular potentials were recorded from the middle and caudal region of the saccule while sound was presented via an underwater speaker. Our results indicate saccular hair cells from reproductive type II males had thresholds based on measures of sound pressure and acceleration (re. $1 \mu \mathrm{Pa}$ and $1 \mathrm{~ms}^{-2}$, respectively) that were $\sim 8-21 \mathrm{~dB}$ lower than non-reproductive type II males across a broad range of frequencies, which include the dominant higher frequencies in type I male vocalizations. This increase in type II
\end{abstract}

A. A. Bhandiwad and E. A. Whitchurch contributed equally.

Joseph A. Sisneros

sisneros@uw.edu

1 Department of Psychology, University of Washington, Seattle, WA 98195-1525, USA

2 Laboratoire de Morphologie Fonctionnelle et Evolutive, Université de Liège, Institut de Chimie, Bât. B6c, Quartier Agora, 4000 Liège, Belgium

3 JASCO Applied Sciences, Silver Springs, MD 20902, USA

4 Department of Biology, University of Washington, Seattle, WA 98195-1800, USA

5 Virginia Merrill Bloedel Hearing Research Center, University of Washington, Seattle, WA 98195-7923, USA auditory sensitivity may potentially facilitate eavesdropping by sneaker males and their assessment of vocal type I males for the selection of cuckoldry sites during the breeding season.

Keywords Hearing - Particle acceleration - Teleost . Saccule $\cdot$ Hair cells

\section{Introduction}

Many teleost fishes use acoustic signals for social communication, and in many species, the use of these social acoustic signals is essential for successful courtship and reproduction (Bass and McKibben 2003; Ladich 2004; Fine and Parmentier 2015). Recent studies have shown that many teleosts possess both behavioral and neural adaptations for the production and perception of acoustic signals that are necessary for reproduction and survival (Bass and McKibben 2003; Bass and Ladich 2008; Kelley and Bass 2010). One vocal teleost that is a good species for investigating neural mechanisms of vocal production and signal perception is the plainfin midshipman fish, Porichthys notatus (Bass et al. 1999; Sisneros 2009a; Forlano et al. 2015). This nocturnally active marine teleost produces a relatively simple repertoire of acoustic signals for intraspecific social communication that includes "grunts", "growls" and "hums" (Bass et al. 1999; Sisneros 2009a). While all three adult sexual phenotypes (female and two male sexual phenotypes: types I and II) are known to produce the short-duration, broadband agonistic "grunts", only type I "singing" males build nests and produce the long-duration, broadband agonistic "growls" and multiharmonic advertisement calls or "hums" during the summer breeding season (Bass and McKibben 2003; Brantley and Bass 1994). 
Previous studies have shown that both the female and type I male auditory systems are seasonally adapted to detect the broadband agonistic calls and the dominant harmonic components of the advertisement calls produced by type I males during the breeding season (Sisneros and Bass 2003; Rohmann and Bass 2011). These adaptations are potentially important for conspecific detection and localization, mate choice decisions and intraspecific male competition (Sisneros 2009a, b).

In contrast to "singing" type I males, "sneaker" type II males employ an alternative reproductive strategy that does not require building a nest or producing an advertisement call to attract mates. Instead, type II males, which have neuroendocrine traits similar to that of females, engage in satellite or sneak-spawning to steal fertilizations from type I males that are actively courting females in the nest (Brantley and Bass 1994). While females and type I males are known to be better suited to detect midshipman vocalizations during the breeding season (Sisneros and Bass 2003; Sisneros 2009b; Rohmann and Bass 2011), the auditory sensitivity of sneaker type II males has yet to be characterized. There is no a priori reason to expect that seasonal plasticity of midshipman auditory sensitivity would be limited to females and type I males.

The sensitivity of auditory systems in fishes can be measured in terms of acoustic particle motion and pressure. All fishes are likely sensitive to the particle motion component of sound via their otolithic end organs which essentially function as inertial accelerometers (de Vries 1950; Fay 1984). This common mode of hearing in fishes enables them to detect particle motion, the directional vector component of sound, as opposed to sound pressure, which is a scalar quantity containing no directional information. Some more recently derived fishes such as Otophysan fishes (e.g., goldfish, carp, zebrafish, etc.) have evolved pressure sensitivity as a result of having a specific connection between the inner ear and an air bubble (e.g., the swim bladder) (Fay and Popper 1980; Blaxter 1981; Schellart and Popper 1992). Recently Popper and Fay (2011) proposed that all future fish hearing studies should independently characterize particle motion and sound pressure sensitivities of fish regardless of any hearing specializations that fish may possess (e.g., accessory hearing structures such as Weberian ossicles that connect the swim bladder to the inner ear). Previous auditory physiology studies of the plainfin midshipman fish have primarily reported auditory responses with regard only to sound pressure (McKibben and Bass 1999, 2001; Sisneros et al. 2004; Sisneros and Bass 2003, 2005; Sisneros 2007, 2009b; Alderks and Sisneros 2011; Rohmann and Bass 2011; but for the exception see Weeg et al. 2002). Thus, better characterization of particle motion sensitivity in this species and across the different sexual phenotypes is warranted.
The primary goal of this study was to characterize auditory-evoked potentials from the saccule in reproductive and non-reproductive type II male midshipman to determine whether this sexual phenotype exhibits seasonal differences in auditory threshold and frequency response of the saccular hair cells to behaviorally relevant auditory stimuli. A secondary goal was to provide a description of the acoustic particle motion sensitivity of the midshipman auditory system that could be used for future comparisons across midshipman sexual phenotypes and with other species. We hypothesized that the auditory saccular sensitivity of type II males during the breeding season would be adapted to detect the dominant frequency content of the type I male vocalizations, which would be beneficial for type II males in their selection of type I male cuckoldry sites for sneak or satellite spawning. We also compare our findings to that of the saccular potentials reported for females (Sisneros 2009b) and type I males (Rohmann and Bass 2011) and interpret our results as they relate to possible auditory adaptations for acoustic communication during the reproductive season.

\section{Materials and methods}

\section{Experimental animals}

Summer reproductive adult type II male midshipman fish (Porichthys notatus) were hand-collected from exposed intertidal nests at low tide in Tomales Bay, CA, USA, during the reproductive season (May-July 2010). Winter nonreproductive adult type II males were collected by otter trawl (RV Kittiwake, Bio-Marine Enterprises) at depths from 70 to $100 \mathrm{~m}$ in the Puget Sound near Edmonds, WA $(47.82 \mathrm{~N}, 122.38 \mathrm{~W})$ and in Shilshole Bay near Ballard, WA $(47.68 \mathrm{~N}, 122.41 \mathrm{~W})$ during the winter months (December-February) of 2007, 2010 and 2016. Note that the limited number of non-reproductive type II males used in this study was sampled over multiple years due to their scarcity during the non-reproductive winter (personal observations, Sisneros). Based on our experience, the collection of type II males during winter trawls in the waters of Puget Sound, WA, is often a rare and serendipitous event.

Type II males were distinguishable from type I males based on their standard body length (SL), gonadosomatic index (GSI) and visual inspection of their genital papilla and their swim bladder sonic muscles. The reproductive type II males used in this study were noted to have pale and underdeveloped sonic muscles instead of the typical large red sonic muscles observed in summer reproductive type I males. In addition, the SL and GSI ranges of type II males reported here were well within the ranges reported for type II males in a previous study (Grober et al. 1994) that were 
collected at the same sites in Tomales Bay, CA. GSI was calculated as $100 \mathrm{x}$ gonad mass/(body mass - gonad mass), based on Tomkins and Simmons (2002). Soon after summer field collection ( $<4-5$ days), type II males were transported to the University of Washington in Seattle, WA, where they were housed in aquaria at $13-15^{\circ} \mathrm{C}$ and kept under reverselight cycle (light:dark-14:10 h). The experiments performed with summer reproductive type II males were conducted during the dark phase of the light/dark cycle since these fish are known to be nocturnally active. Animals were hand-fed defrosted shrimp and/or live feeder guppies two to three times a week. Auditory physiology recordings and measurements were made within 26 of collection for most animals except for two non-reproductive type II males that were recorded approximately a few months after collection while being maintained under a short-day winter photoperiod ( $8 \mathrm{~h}$ light, $16 \mathrm{~h}$ dark). A simple linear regression between gonadosomatic index (GSI) and days in captivity was performed to test for time-dependent changes in breeding state, with the regression coefficient $\left(r^{2}\right)$ reported.

\section{Experimental procedures}

The procedures for exposing and recording from the inner ear saccule followed those used in previous studies (Sisneros 2007, 2009b; Alderks and Sisneros 2011). Briefly, animals were initially anesthetized in a $0.025 \%$ ethyl- $p$-aminobenzoate/saltwater bath until the fish's opercula ceased movement for at least $5 \mathrm{~min}$. The animals were then immobilized with an intramuscular injection of pancuronium bromide (approx. $0.5 \mu \mathrm{g} \mathrm{g}^{-1}$ body mass). Skin, muscle and bone were removed just dorsal to the otic capsules, and a 2.5-cm hydrophobic dam was built around the craniotomy to enable submersion without exposing the brain and inner ear to salt water. The cranial cavity was then filled with Teleost Ringer's solution to prevent drying. A subcutaneous injection of $0.25 \%$ bupivacaine (approx. $1 \mu \mathrm{g} \mathrm{g}^{-1}$ body weight) was administered around the surgical site as an analgesic. During the experiment, the animals were submerged underwater such that the saccules were approximately $3 \mathrm{~cm}$ below the water surface while fresh saltwater was circulated over the gills. The craniotomy was periodically checked visually to verify stable blood flow in the brain and inner ear organs.

Animals were suspended by a custom-built acrylic stereotaxic head-holder that was positioned $10 \mathrm{~cm}$ above an underwater speaker (UW-30, Telex Communications, Burnsville, MN, USA) in the center of a 40-cm-diameter Nalgene recording tank. The bottom of the tank contained an approximately $4.5 \mathrm{~cm}$ layer of small rock substrate in which the speaker was embedded so that only the top $2 \mathrm{~cm}$ of the speaker projected upwards into the water column. The water was initially chilled to $12^{\circ} \mathrm{C}$ and maintained between 14 and $15^{\circ} \mathrm{C}$ for the duration of the recording session. The distance from the water surface to the surface of the speaker was $13 \mathrm{~cm}$. The tank was positioned on an inflated pneumatic table, inside an acoustic isolation chamber (Industrial Acoustics, New York, NY, USA), and all recording equipment were housed outside this chamber.

Saccular potentials were recorded using glass electrodes (1.0-4.0 M $\Omega$ ) filled with a $3 \mathrm{M} \mathrm{KCl}$ solution. The electrodes were visually positioned in the space between the otolith and sensory maculae in the middle/caudal region of the saccule. Both left and right saccules were used in this study. Recording fidelity was assessed by comparing the magnitude of the saccular potentials recorded during the blank (no stimulus presented) and auditory stimulus test trials. The auditory stimuli were presented were of equal amplitude (with $\pm 1-2 \mathrm{~dB}$ ) at a given sound level across all frequencies tested. Electrode signals were band-pass-filtered (130-3000 Hz, Stanford Research Systems SR 650), amplified by $100 \times$ (Getting 5A), and then sent to a lock-in amplifier (Stanford Research Systems SR 830) and digitized at $20 \mathrm{kHz}$ (Micro1401 mkII, Cambridge Electronic Design). Data acquisition and stimulus timing were controlled by a custom MATLAB (MathWorks Inc., Natick, MA) script. The output of the lock-in amplifier reflects the relative amplitude of the inner ear saccule's hair cell response to a tonal stimulus. Because the opposing hair cell orientations within the saccular sensory maculae result in a double-frequency response, the saccular potential is defined here as the amplitude the hair cell response at the second harmonic of the stimulus (Cohen and Winn 1967; Sisneros 2007).

\section{Acoustic stimulus generation and calibration}

Single tones were presented at $75-85 \mathrm{~Hz}$ in $10 \mathrm{~Hz}$ steps and $105-385 \mathrm{~Hz}$ in $20 \mathrm{~Hz}$ steps. Stimuli were generated by the lock-in amplifier, amplified by an audio amplifier, and then played through the underwater speaker. Stimuli were $750 \mathrm{~ms}$ in length and separated by a 500-ms interval. Lock-in measurements were sampled during the last $20 \mathrm{~ms}$ of the stimulus. This data collection procedure and use of the lock-in amplifier has been shown to be very sensitive and effective in measuring the saccular potentials at the reported frequencies (Sisneros 2007, 2009b; Rohmann and Bass 2011; Vasconcelos et al. 2011). Each recording session began with control trials (no auditory stimulus) followed by stimulus test trials in which auditory frequency stimuli at a given sound level were presented in a pseudorandom order.

Calibrations were performed before every experiment, after the tank water was initially cooled, but before the fish was placed in the tank. During calibration of the auditory stimuli, the hydrophone and accelerometer were centered 
$10.5 \mathrm{~cm}$ above the speaker $(2.5 \mathrm{~cm}$ below water surface $)$ at the position of the fish's ear. Stimuli were equalized in SPL using an iterative MATLAB script that measured power spectral density for each frequency from recordings taken through a mini-hydrophone (model 8103, Bruel and Kjaer). At each iteration, the voltage signal sent to the speaker was scaled until the measured SPL output at each frequency was within $0.5 \mathrm{~dB}$ of the desired amplitude $(130 \mathrm{~dB}$ re.: $1 \mu \mathrm{Pa})$. This SPL level was chosen for calibration because $130 \mathrm{~dB}$ SPL is well above the noise floor for our measurement devices, and it is consistent with sound levels for type I male midshipman calls within and near their nests in the field and therefore within the range of stimulus amplitudes tested here (Bass and Clark 2003). The frequency stimuli used in this study were based on that used in previous studies (Sisneros and Bass 2003; Sisneros 2007, 2009b; Rohmann and Bass 2011). The intentional of this study was to compare the saccular sensitivity of type II male $P$. notatus across season (non-reproductive vs. reproductive state) and compare the saccular sensitivity with that of females (Sisneros 2009b) and type I males (Rohmann and Bass 2011) under similar experimental conditions. The data presented in sound pressure and particle acceleration to describe auditory sensitivities should not be considered in terms of absolute valve given the constrains of our experimental tank setup, but instead should be used as a means to make quantifiable comparisons of the relative seasonal, reproductive state-dependent differences in auditory saccular sensitivity across the three sexual phenotypes (females, and type I and II males).

Consistent with previous studies, acceleration measurements were collected in three dimensions relative to fish orientation inside the tank: $x$ (anterior/posterior), $y$ (left/right) and $z$ (dorsal/ventral) (Casper and Mann 2006; Horodysky et al. 2008; Wysocki et al. 2009) using a custom-modified three-dimensional underwater accelerometer (PCB model VW356A12, sensitivity $=100 \mathrm{mV} \mathrm{g}^{-1}$ (10.2 $\mathrm{mV} \mathrm{ms}^{-2}$ ) PCB Piezotronics, Depew, NY) that was encased in syntactic foam and epoxy to make it neutrally buoyant. We characterized the acoustic impedance (ratio of sound pressure to particle velocity) of our experimental tank conditions as suggested by Popper and Fay (2011), using the calibration measurements at different three SPLs: 118, 130, and $145 \mathrm{~dB}$ (re.: $1 \mu \mathrm{Pa}$ ). Figure 1a shows the
Fig. 1 Acoustic characterization of the tank used in this study. a Acoustic impedance, calculated as the ratio of sound pressure level to particle motion level, measured in the frequency ranges tested at three sound pressure levels $(118,130$ and $145 \mathrm{~dB}$ re. $1 \mu \mathrm{Pa}$ ). Note that the three sound pressure levels tested are parallel and show no major resonances at any of the tested frequencies. b Amplitude profiles of three sound pressure levels $(118,130$ and $145 \mathrm{~dB}$ re. $1 \mu \mathrm{Pa})$ are relatively constant and frequency-invariant. c Particle acceleration levels measured at $130 \mathrm{~dB}$ re. $1 \mu \mathrm{Pa}$ at each of the frequencies used in this study in the three Cartesian axes $[x, y, z]$, represented by pink, blue and red, respectively. The majority of the energy is contained in the dorsoventral (" $z$ ") axis (red bars)
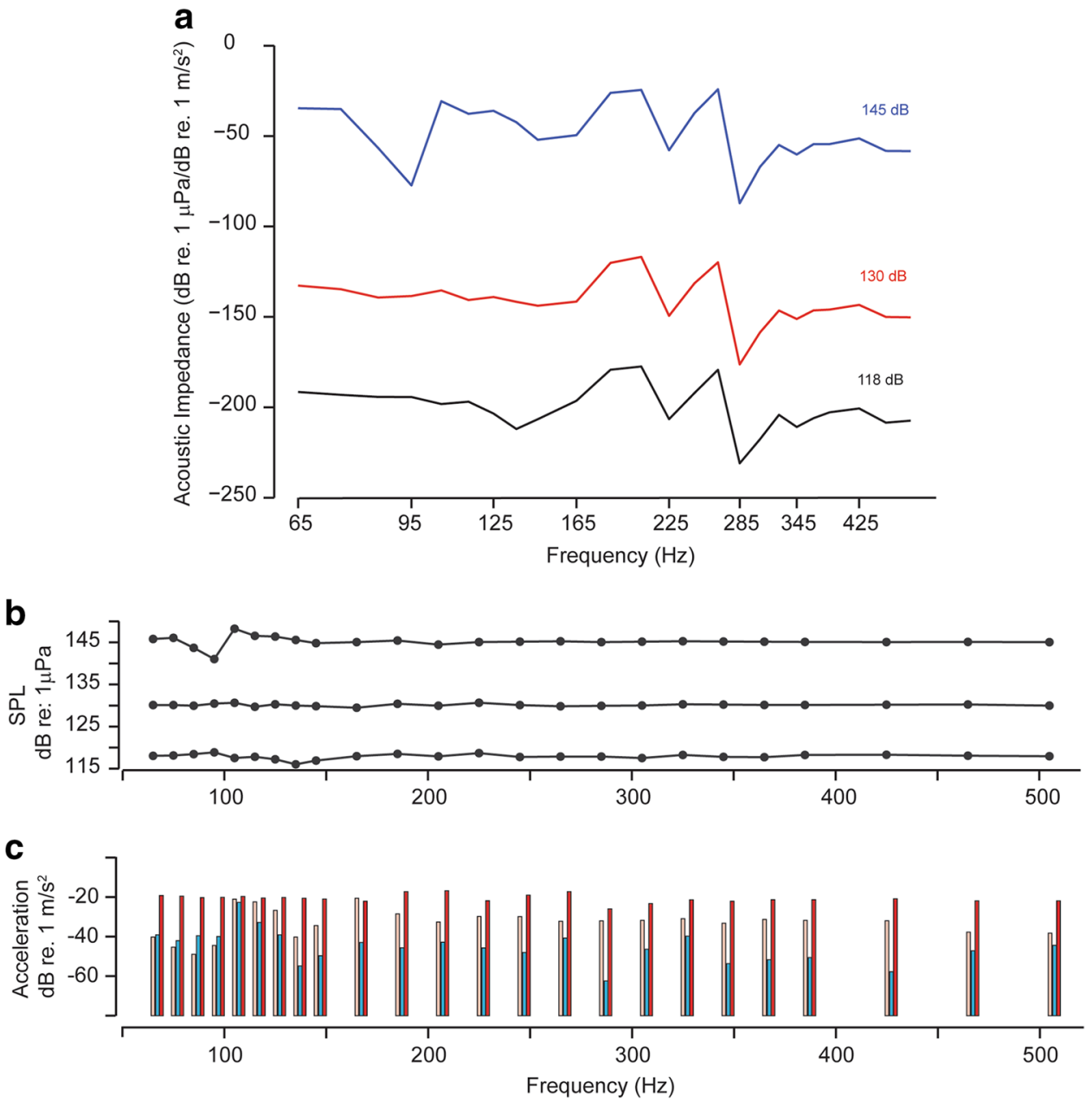
ratio of sound pressure level to particle velocity for each frequency recorded in our experimental tank setup. The calibrated sound pressure levels were relatively constant across the test frequencies regardless of amplitude (e.g., see Fig. 1b which shows stimulus calibrations at 118, 130 and $145 \mathrm{~dB}$ re. $1 \mu \mathrm{Pa}$ ). For any given sound level, particle acceleration was relatively constant and greatest in the $z$ dimension relative to the dimensions ( $x$ and $y$ ) orthogonal to speaker motion (Fig. 1c) and particle acceleration in all three dimensions scaled linearly across the SPLs tested.

\section{Data analyses}

The acoustic stimuli and the evoked saccular potentials were measured simultaneously and sampled at $20 \mathrm{kHz}$. During the recordings, the hydrophone and accelerometer were placed approximately $13 \mathrm{~cm}$ from the center of the tank (where the fish was positioned) at a depth of $10 \mathrm{~cm}$.
Representative examples of a control (no auditory stimulus) and test trial $(75 \mathrm{~Hz}$ at $130 \mathrm{~dB})$ are shown in Fig. 2. The middle $700 \mathrm{~ms}$ of the recorded signals was used to calculate the relative power of each frequency using the fast Fourier transform ("FFT," MATLAB version 2007). Note that any acoustic reflections of the stimulus from the tank walls and water surface did not alter the sound pressure waveform of the acoustic signal, even at the lowest frequencies tested (e.g., $75 \mathrm{~Hz}$ ) as shown in Fig. 2. There was no significant background noise measured by the hydrophone or accelerometer during the control trials (note gray traces in Fig. 2). Saccular potentials contained only relative small peaks of noise at the harmonics of $60 \mathrm{~Hz}$ during the recordings of the controls. During test trials, the saccular potentials contained significant energy at the second harmonic of the stimulus frequencies (Fig. 2d). Only the second harmonic component was used by the lock-in amplifier to determine the saccular potential. a Hydrophone
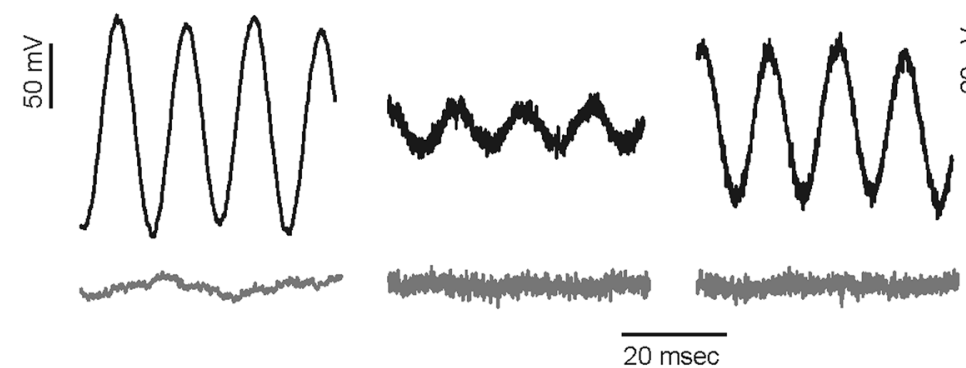

C
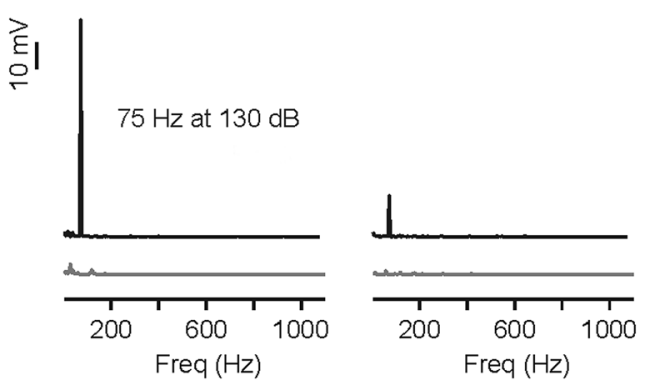

Fig. 2 Representative examples of the acoustic stimulus and the evoked saccular potentials recorded from type II male $P$. notatus that were simultaneously measured during physiology experiments. a During the experiments, the fish was positioned in the center of the tank and the hydrophone and/or accelerometer was positioned at a depth of $10 \mathrm{~cm}$ and placed horizontally approximately two-third of the distance from the center to the rim of the experimental tank [black trace shows recorded signal during stimulus presentation while gray trace shows the absence of signal during control trial (no auditory stimulus); note only two of the three recorded accelerometer axes are shown here $-X$ (anterior/posterior) and $Y$ (dorsal/ventral)]. b During stimulus presentation, the saccular potentials were evoked during the duration of stimulation [black trace shows the recorded evoked saccular potential from the recording electrode during a stimulus presentation of $75 \mathrm{~Hz}$ at $130 \mathrm{~dB}$ re: $1 \mu \mathrm{Pa}$, while the gray trace
Z-Dorsal/Ventral

b Electrode
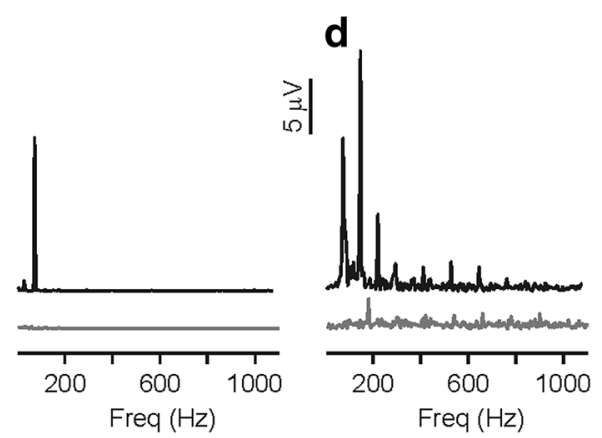

shows only background noise during a control (no auditory stimulus) trial]. c Power spectrum of the auditory stimulus as measured by the hydrophone and accelerometer shown in a. The corresponding power spectra are shown below the measurement for the hydrophone and the recorded $X$ and $Z$ accelerometer axis [black trace is the power spectra for the stimulus frequency of $75 \mathrm{~Hz}$ at $130 \mathrm{~dB}$ re: $1 \mu \mathrm{Pa}$, while the gray trace shows power spectra for control trial (no auditory stimulus)]. d Power spectrum of the recorded evoked saccular potential shown in b black trace is the power spectra of the saccular potentials during auditory stimulation while gray trace shows power spectra of saccular potentials during control (no auditory stimulus) trial. Note that the greatest energy in the power spectra of the evoked saccular potentials occurs at the 2 nd harmonic of the stimulus frequency (the expected response from opposite oriented hair cells in the saccule) 
The threshold of the evoked saccular response was determined using: (1) the average potential recorded in the absence of a stimulus plus two standard deviations and (2) a continuous estimate of the saccular responses $\left(y_{\text {est }}\right)$ based on the recorded evoked responses near-threshold levels, which was generated by fitting the data with an exponential function (see Fig. 3, thick fitted line using the equation below). Auditory thresholds were determined using exponential rate-level functions that were fitted to the records of the evoked potentials and then compared to the saccular potential recorded in the absence of an auditory stimulus.
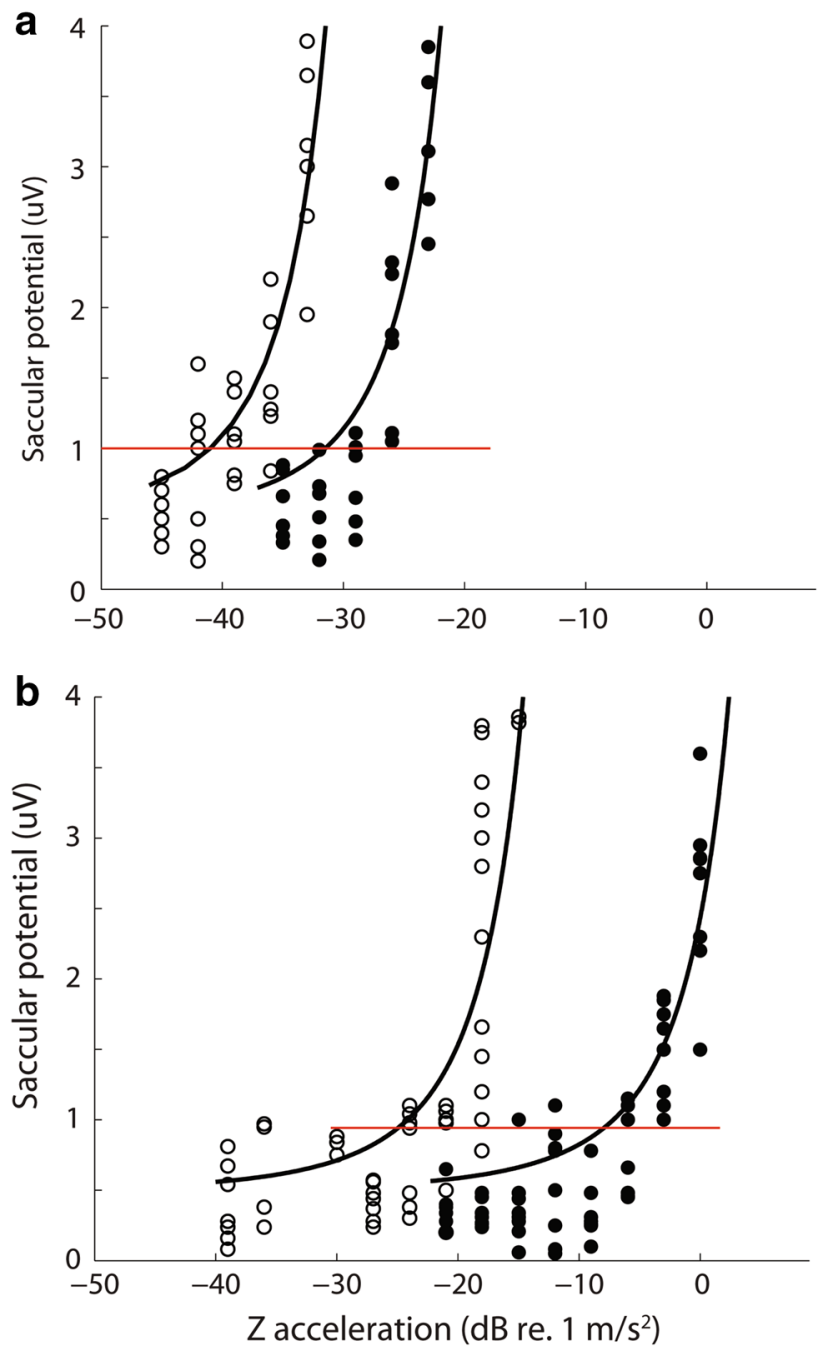

Fig. 3 Illustrated examples of how the evoked saccular potential thresholds were determined at the tested frequencies. Figure shows examples at the lowest frequency tested (i.e., $75 \mathrm{~Hz}$ ) (a) and the highest frequency tested (i.e., $385 \mathrm{~Hz}$ ) (b) for reproductive (open circles) and non-reproductive fish (filled circles). Each data set was fitted with a best-fit model curve (black lines) and thresholds were interpolated from the best-fit curve (see detailed description in "Materials and methods"). Auditory threshold level was calculated as two standard deviations above baseline and is represented in figure as the horizontal red line
Figure 3 shows two illustrated examples of threshold determination using the rate-level function near threshold at the minimum and maximum frequencies tested. Auditory threshold was defined as the minimum stimulus level above which the estimated saccular response $\left(y_{\text {est }}\right)$ exceeded 2 standard deviations above the recorded evoked potential in the absence of a stimulus (Fig. 3 horizontal line). Exponentials were fit using data points from near-threshold trials (10-15 dB above and below threshold) using the following equation:

$y_{\text {est }}=\frac{\mathrm{e}^{x * b_{1}}}{1+\mathrm{e}^{x * b_{2}}}$,

where $x$ is the stimulus amplitude, $y_{\text {est }}$ is the estimated saccular response, and $b_{1}$ and $b_{2}$ are the fitted variables defining the curve. Particle motion thresholds were derived from sound pressure thresholds using best-fit linear coefficients derived from calibrations. Because there was a linear relationship between pressure and particle motion at all frequencies tested, best-fit linear transformations were derived for each frequency using pressure calibration measurements and the equivalent particle motion measurements in the $x, y$ and $z$ axes. After deriving thresholds in the $x$, $y$ and $z$ dimensions, particle motion threshold reported as the combined magnitude vector, calculated as $20 \mathrm{log}$ $\left(\sqrt{ }\left(x^{2}+y^{2}+z^{2}\right)\right)$ similar to Wysocki et al. (2009) and Vasconcelos et al. (2011).

The overall effect of reproductive state on the auditory saccular thresholds based on sound pressure and particle acceleration in non-reproductive and reproductive type II male midshipman was analyzed using a repeated measures ANOVA with thresholds for each of the 17 frequencies tested $(75-385 \mathrm{~Hz})$ as the repeats (i.e., within-subject factors) and reproductive state of the animal as the betweensubject factor followed by the HSD test with unequal $N$ for post hoc planned comparisons of thresholds at each stimulus frequency. For all statistical tests, $\alpha$ was set at 0.05 . Statistical analyses were performed using Statistica for Windows (StatSoft).

\section{Results}

Evoked saccular potentials were recorded from a total of 32 adult type II male midshipman: 5 non-reproductive type II males with a size range of 10.2-16.0 cm $\mathrm{SL}$ [mean $\mathrm{SL}=13.5 \pm 1.8 \mathrm{~cm} \mathrm{SD}$, mean body mass $(\mathrm{BM})=35.2 \pm 17.1 \mathrm{~g} \mathrm{SD}$, and mean $\mathrm{GSI}=4.4 \pm 3.1 \mathrm{SD}]$ and 32 reproductive type II males with a size range of 8.0-10.5 cm SL [mean SL $=9.5 \pm 0.7 \mathrm{~cm} \mathrm{SD}$, mean body mass $(\mathrm{BM})=10.4 \pm 2.3 \mathrm{~g} \mathrm{SD}$, and mean $\mathrm{GSI}=13.5 \pm 4.5$ $\mathrm{SD}$. The evoked saccular potentials were recorded from all type II males within 26 days of field collection, with 
the exception of two non-reproductive type II males that were recorded approximately 5-7 months after collection; however, both of these males were maintained on a shortday "winter" photoperiod. No relationship was observed between GSI and the number of days that the animal were kept in captivity within 26 days of collection $\left(r^{2}=0.047\right.$, $p>0.2$ ).

Auditory thresholds based on sound pressure and particle acceleration were determined for whole populations of hair cells in the saccule of non-reproductive and reproductive type II males. Threshold tuning curves were constructed using an exponential rate-level function that was fitted to the records of evoked saccular potentials and compared to the evoked potentials recorded in the absence of a stimulus (background noise measurement) (Fig. 3). The auditory threshold at each stimulus frequency was designated as the lowest stimulus level that evoked a saccular potential greater than $2 \mathrm{SD}$ above the background noise measurement (Fig. 3, red line). The auditory thresholds for the recorded saccular hair cells based on sound pressure and particle acceleration are summarized in Fig. 4.

The threshold tuning curves based on sound pressure generally consisted of tuning profiles with lowest thresholds at 75 and $85 \mathrm{~Hz}$ that gradually increased to highest thresholds at frequencies $\geq 205 \mathrm{~Hz}$ (Fig. 4a). Significant pressure threshold differences between non-reproductive and reproductive type II males were observed at every tested frequency except $75 \mathrm{~Hz}$ (repeated measures ANOVA: between-subject factor reproductive state, HSD test with unequal $\left.n: F_{(17,21)}=4.94, p<0.001\right)$. The auditory thresholds of the saccular hair cells from reproductive male type II were $\sim 8-20 \mathrm{~dB}$ (re. $1 \mu \mathrm{Pa}$ ) lower than non-reproductive type II males at frequencies from 85 to $385 \mathrm{~Hz}$ (Fig. 4a).

The threshold tuning curves based on particle acceleration were similar in shape to the pressure threshold profiles with lowest thresholds at 75 and $85 \mathrm{~Hz}$ that gradually increased to highest thresholds at frequencies $\geq 185 \mathrm{~Hz}$ (Fig. 4b). Significant threshold differences based on acceleration between non-reproductive and reproductive type II males were observed at every frequency except $75 \mathrm{~Hz}$ (repeated measures ANOVA: between-subject factor reproductive state, HSD test with unequal $n: F_{(17,21)}=4.95$, $p<0.001)$. The auditory acceleration thresholds of the saccular hair cells from reproductive male type II were $\sim 8-20 \mathrm{~dB}$ re. $1 \mathrm{~m} \mathrm{~s}^{-2}$ lower than non-reproductive type II males at frequencies from 85 to $385 \mathrm{~Hz}$ (Fig. 4b).

\section{Discussion}

The goal of this study was to characterize the evoked saccular potentials of type II male midshipman to determine whether auditory threshold and frequency response of
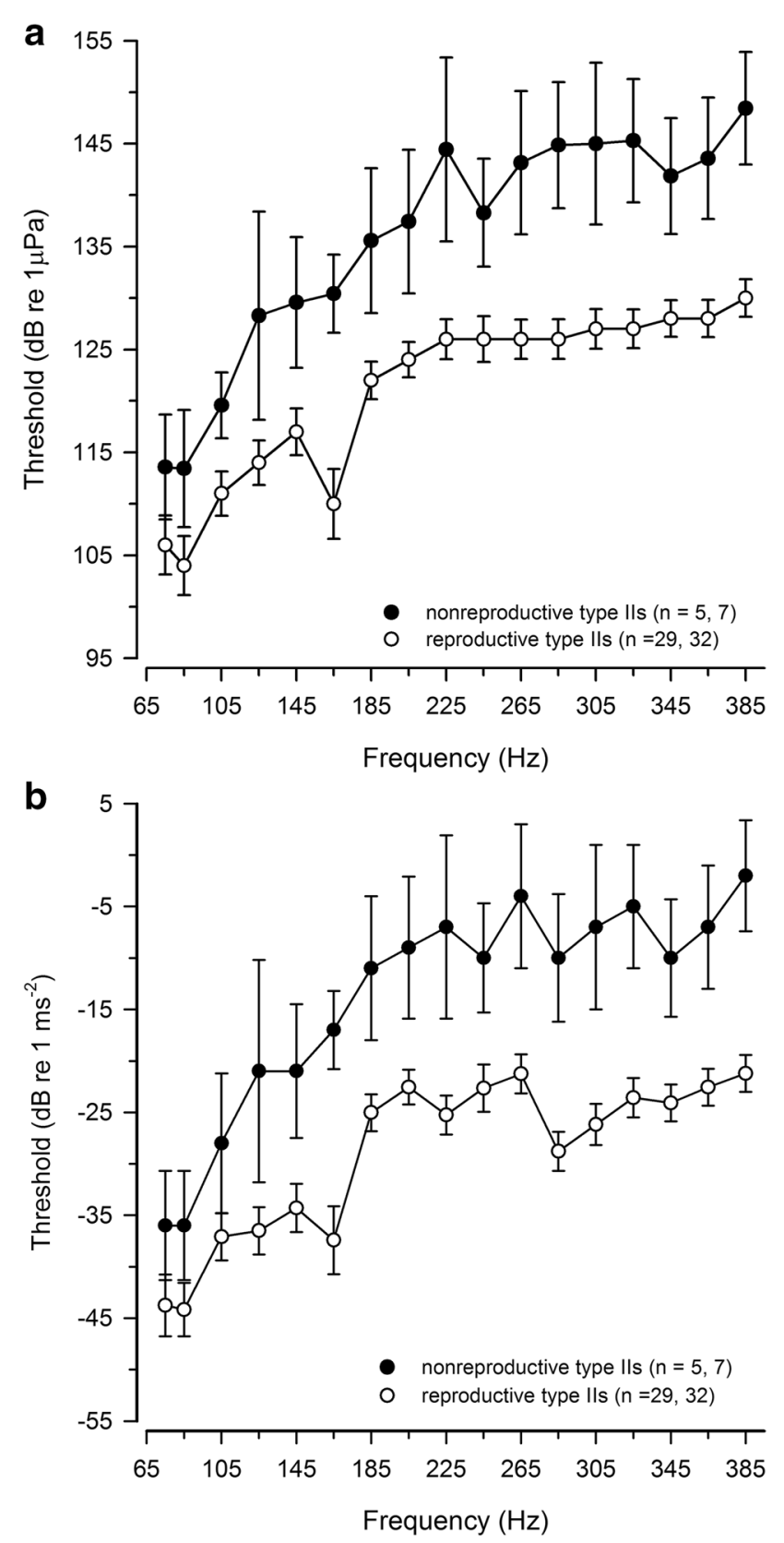

Fig. 4 Seasonal changes in saccular sensitivity-based sound pressure and particle acceleration measurements in the plainfin midshipman fish (Porichthys notatus). a Auditory threshold tuning curves for non-reproductive and reproductive type II males based on saccular potential recordings and measurements of sound pressure. All data are plotted as mean $\pm 95 \%$ confidence limit. The number of animals and records are indicated in parentheses. b Auditory threshold tuning curves for non-reproductive and reproductive type II males based on saccular potential recordings and measurements of acoustic particle acceleration. All data are plotted as mean $\pm 95 \%$ confidence limit. The number of animals and records are indicated in parentheses

saccular hair cells change seasonally to behaviorally relevant auditory stimuli. Our results indicate that the summer reproductive type II males were more sensitive than winter 
non-reproductive type II males across a range of frequencies tested $(85-385 \mathrm{~Hz})$. Our data support the hypothesis that the saccular sensitivity of reproductive type II males is similar in sensitivity to the other reproductive sexual phenotypes (females and type I males) and better adapted than that of non-reproductive type II males to detect the dominant frequency content of the type I male vocalizations. In this discussion, we also interpret our results as they relate to the reception of social acoustic signals based on sound pressure and particle motion and how the changes in auditory sensitivity of type II males may facilitate the localization and assessment of midshipman social acoustic signals during the breeding season.

\section{Appropriate stimuli}

There has been much discussion regarding the appropriate sound stimuli to measure in fish hearing studies (Popper and Fay 1993, 2011; Fay and Popper 2012). Sound can be measured in terms of both acoustic particle motion and pressure. Fish have evolved at least two modes of hearing: an inertial (particle motion) mode and a pressure-mediated mode. All teleost fishes are thought to be capable of detecting a particle motion vector, such as acoustic particle acceleration, via their inner otolithic end organs which essentially act as acceleration detectors that respond directly to displacement of the fish by particle motion (De Vries 1950; Hawkins 1993; Sisneros and Rogers 2016). In contrast, the pressure-mediated mode of fish hearing requires the use of specialized morphological adaptations that enable fish to transduce the pressure-induced vibrations of the swim bladder to the otolithic end organs to detect sound pressure. Otophysan fish, such as the goldfish (Carassius auratus) and zebrafish (Danio rerio), possess skeletal adaptations known as Weberian ossicles that link the swim bladder to the inner ear for enhanced pressure detection while other fishes such the Hawaiian squirrelfish (Myripristis kuntee) and the West African ladyfish (Elops lacerta) have no specialized connections between the swim bladder and inner ear, but do exhibit increased pressure sensitivity simply due to the close proximity of these gas-filled structures to the inner ear (Greenwood 1970; Coombs and Popper 1979; Braun and Grande 2008; Tricas and Boyle 2015). Additional studies have shown that natural or artificial gasfilled bladders without direct apposition to the inner ear can also significantly enhance hearing sensitivity in fishes (Chapman and Sand 1974; Jerko et al. 1989). Given that the swim bladder in female and type II male midshipman extends anteriorly via "horn-like" extensions of the swim bladder to caudal regions of the otic capsule (Whitchurch et al. 2012), and that this caudal region of otic capsule has been proposed to be an "acoustic channel" to the saccule through the neurocranium (Edds-Walton et al. 2015), the midshipman is very likely sensitive to both sound pressure and particle motion. Thus, in the current study we characterized the midshipman auditory thresholds in terms of both sound pressure and particle motion. As suggested by Popper and Fay (2011), we also report the impedance (ratio of pressure to particle velocity) of our test tank (see Fig. 1a) so that the acoustic impedance of the tank conditions in this study can be used in comparison with that of future fish hearing studies.

\section{Reproductive state-dependent changes in auditory saccular sensitivity}

We observed dramatic reproductive state-dependent differences in the sound pressure and particle acceleration thresholds of the saccule in type II males. The relative differences in sensitivity between reproductive and nonreproductive type II males for both pressure and acceleration (re. $1 \mu \mathrm{Pa}$ and $1 \mathrm{~ms}^{-2}$, respectively) varied between 8 and $21 \mathrm{~dB}$ at frequencies from 75 to $385 \mathrm{~Hz}$ (Fig. 4). Furthermore, the saccular thresholds of reproductive type II males were at least $13 \mathrm{~dB}$ lower (an enhanced sensitivity equivalent to 4.5 times greater) than non-reproductive type II males at frequencies $>185 \mathrm{~Hz}$, which corresponds to the higher frequency components with the majority of energy in type I male vocalizations (Fig. 5). Nesting type I males produce three types of acoustic signals that include agonistic grunts and growls and advertisement calls known as hums (Bass et al. 1999; Forlano et al. 2015). Reproductive type I males often produce trains of broadband, short-duration (50-200 ms) grunts (Fig. 5a) to fend or ward off potential nest intruders (Brantley and Bass 1994). Grunts trains produced by type I males can have a repetition rate of about $2.5 \mathrm{~Hz}$ with grunt intervals of approximately $400 \mathrm{~ms}$ (Bass et al. 1999). Growls are relatively long-duration ( $>1 \mathrm{~s}$ ), multiharmonic signals (Fig. 5b) with fundamental frequencies that gradually change over the duration of the call from 116 to $59 \mathrm{~Hz}$ (Bass et al. 1999). In comparison, the hum is a long-duration, multiharmonic signal with a fundamental frequency that can range from 80 to $102 \mathrm{~Hz}$ (personal observation, JAS; also see Bass et al. 1999). In addition to the fundamental frequency, the hum also contains a number of prominent harmonics that range up to $400 \mathrm{~Hz}$ (Fig. 5c) with additional lower amplitude harmonics ranging up to $800 \mathrm{~Hz}$ and beyond (Bass et al. 1999). In the context of signal recognition and preference, type II males have been observed to exhibit positive phonotaxis to the playback of simulated hums (McKibben and Bass 1998). Our results suggest that the saccular sensitivity of reproductive type II males is well suited to encode frequencies $\geq 165 \mathrm{~Hz}$ which include the dominant higher frequency components in type I male vocalizations including hums (Fig. 5). This enhanced saccular sensitivity may be adaptive and function 
Fig. 5 Comparison between the vocal characteristics of type I male vocalizations and the change in auditory saccular tuning of type II males based on seasonal reproductive state. a Sensitivity increase in auditory saccular thresholds (left y axis) of reproductive type II males compared to non-reproductive type II males at each frequency tested and the power spectrum of a type I male grunt (right y axis, in relative $\mathrm{dB}$ values), inset: the temporal waveform of a type I male grunt recorded at $13.5^{\circ} \mathrm{C}$ in a laboratory setting (scale bar $20 \mathrm{~ms}$ ). b Sensitivity increase in auditory saccular thresholds (left y axis) of reproductive type II males compared to non-reproductive type II males at each frequency tested and the power spectrum of a type I male growl (right $y$ axis, in relative $\mathrm{dB}$ values), inset: the temporal waveform of a type I male growl recorded at $13.5^{\circ} \mathrm{C}$ (scale bar $20 \mathrm{~ms}$ ). c Sensitivity increase in auditory saccular thresholds (left y axis) of reproductive type II males compared to non-reproductive type II males at each frequency tested and the power spectrum of a nesting type I male advertisement call or "hum" (right y axis, in relative dB values), inset: the temporal waveform of a type I male hum recorded at $13.5^{\circ} \mathrm{C}$ at Seal Rock, WA, in a nesting site (scale bar $20 \mathrm{~ms}$ )

to increase the probability of signal detection by sneaker males in shallow water environments where the propagation of acoustic signals is greatly affected by substrate composition and water depth (Rogers and Cox 1988; Bass and Clark 2003; Maruska and Sisneros 2015). The relative high frequencies (approximately those greater than $180 \mathrm{~Hz}$ ) contained with type I vocalizations will propagate further than the calls' fundamental frequencies in shallow water due to the inverse relationship between water depth and the cutoff frequency of sound transmission (Fine and Lenhardt 1983; Rogers and Cox 1988; Bass and Clark 2003). In addition, the substrate composition (e.g., the rocky substrate of the intertidal zone where midshipman breed) can greatly influence the speed of sound in the bottom substrate and affect the cutoff frequency of signal propagation which can attenuate the transmission of low-frequency acoustic signals (e.g., the fundamental frequencies of type I male vocalizations) (Rogers and Cox 1988). Thus, the enhanced sensitivity of type II males to the dominant higher frequencies within type I male vocalizations may potentially facilitate eavesdropping by sneaker males for the assessment of type I male vocalizations and the selection of cuckoldry sites during the breeding season.

The observed seasonal, reproductive state-dependent changes in auditory saccular sensitivity based on sound pressure for type II males were remarkably similar to that reported for female and type I male midshipman. Previously, seasonal and reproductive state-dependent changes in auditory saccular sensitivity based on sound pressure have been reported for both females and type I males across the same range of frequencies from 75 to $385 \mathrm{~Hz}$ (Sisneros 2009b; Rohmann and Bass 2011). Results from this study revealed that the difference in saccular sensitivity based on sound pressure between reproductive and non-reproductive type II males ranged from 9 to $21 \mathrm{~dB}$ at 75 to $385 \mathrm{~Hz}$, respectively (Fig. 4), which is similar to the reproductive

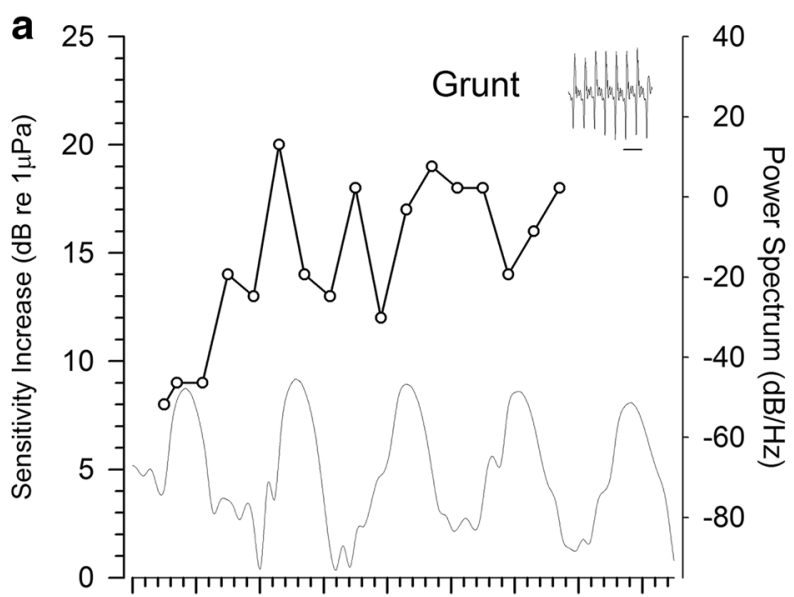

50100150200250300350400450

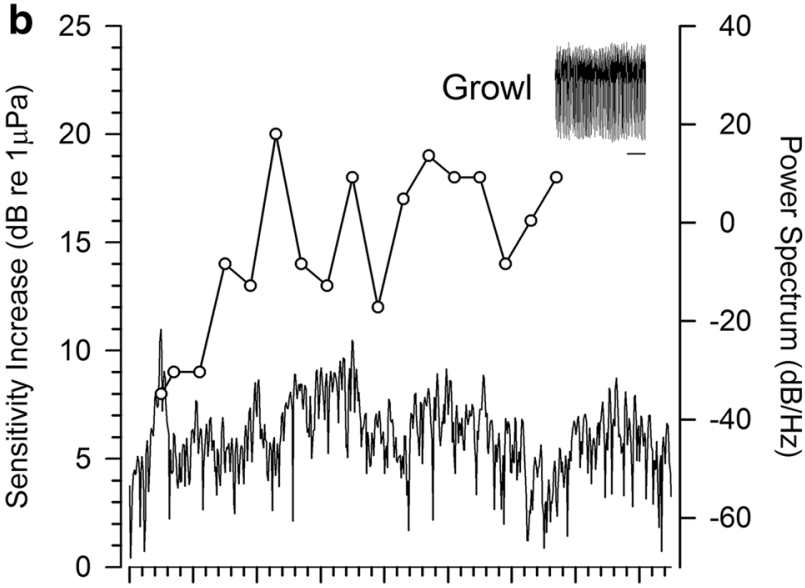

50100150200250300350400450

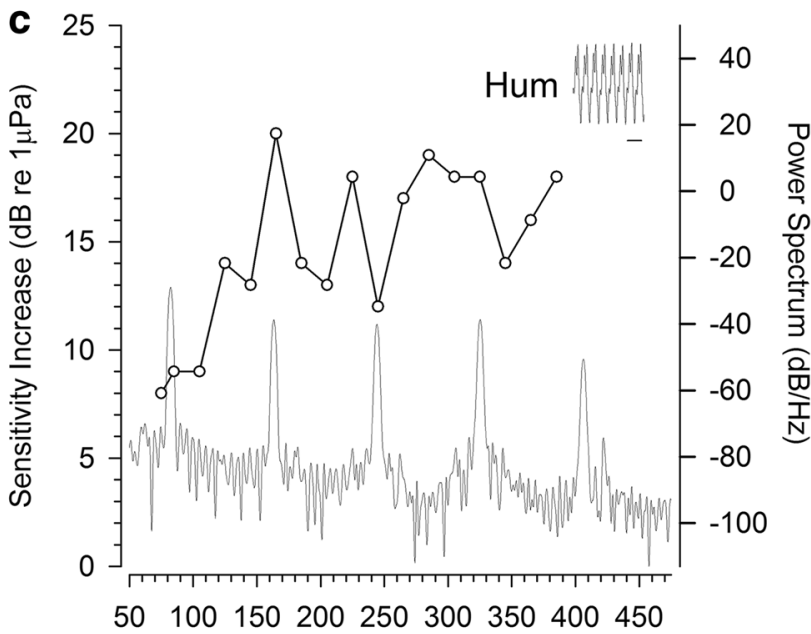

Frequency $(\mathrm{Hz})$

state-dependent differences observed for females and type I males (Fig. 6). Although the sound pressure tuning curves were similar for all three sexual phenotypes, there appear to be slight seasonal differences in the mean threshold profiles between the reproductive and non-reproductive sexual phenotypes. Here, we show that reproductive state-dependent 


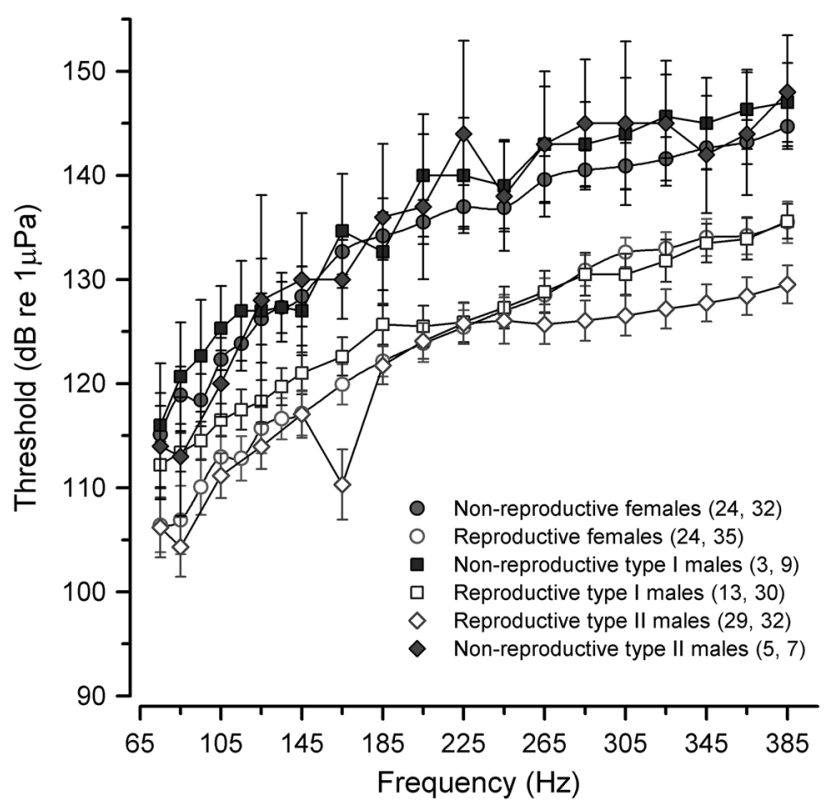

Fig. 6 Frequency sensitivity of saccular hair cells in the plainfin midshipman fish (Porichthys notatus). Shown here is a plot of the auditory saccular tuning curves based on evoked potential recordings in all three adult sexual phenotypes (females, type I and type II males) in reproductive and non-reproductive conditions. All data are plotted as mean $\pm 95 \%$ confidence limit. The number of animals and records are indicated in parentheses. Auditory saccular tuning data for females is adapted from Sisneros (2009b), and from Rohmann and Bass (2011) for type I males

changes in type II male saccular sensitivity were frequency dependent with seasonal threshold differences greatest at frequencies $\geq 125 \mathrm{~Hz} \quad(12-20 \mathrm{~dB})$ and lowest threshold differences at frequencies $<125 \mathrm{~Hz}(\sim 8-9 \mathrm{~dB})$. Similarly, type I males also exhibited reproductive state-dependent changes in saccular sensitivity that were frequency dependent with greatest threshold differences at frequencies $>145 \mathrm{~Hz}(\sim 12-15 \mathrm{~dB})$ and lowest threshold differences at frequencies $\leq 145 \mathrm{~Hz}(\sim 4-9 \mathrm{~dB}$; see Rohmann and Bass 2011). In contrast, seasonal differences in female saccular sensitivity were not frequency dependent but were instead relatively constant $(\sim 8-13 \mathrm{~dB})$ across the same range of tested frequencies from 75 to $385 \mathrm{~Hz}$ (Sisneros 2009b). Consistent across phenotypes is the observation that reproductive midshipman showed significantly lower saccular thresholds than non-reproductive midshipman (Fig. 6). In sum, the results from this study now confirm that seasonal, reproductive state-dependent changes in auditory saccular sensitivity are a species-typical trait found in all midshipman sexual phenotypes.

Although no previous study has examined seasonal differences in acoustic acceleration sensitivity of the saccule in type II males, our results suggest that the saccular acceleration sensitivity of non-reproductive type II males is at least similar to that of non-reproductive type I males
(Weeg et al. 2002). Estimates for particle motion sensitivity in non-reproductive type I male saccular afferents range from -15 to $26 \mathrm{~dB}$ (re. $1 \mathrm{~nm}$ ), which corresponds to a displacement of $0.8-20 \mathrm{~nm}$, whereas our estimate for particle acceleration threshold at the most sensitive frequency tested $(85 \mathrm{~Hz})$ is $-45 \mathrm{~dB} \pm 7 \mathrm{~dB}$ (re. $1 \mathrm{~m} \mathrm{~s}^{-2}$ ). Numerical integration of the mean acceleration threshold value at $85 \mathrm{~Hz}$ provides a rough estimate of a displacement threshold of $17 \mathrm{~nm}$, which is within the displacement sensitivity range reported for type I males by Weeg et al. (2002). Furthermore, the study by Weeg et al. (2002) demonstrated that type I male saccular afferents responded best at 50 and $100 \mathrm{~Hz}$. Although we did not test the same frequencies, we do show that non-reproductive type II males share a similar particle acceleration sensitivity to that of type I males with lowest acceleration thresholds reported at frequencies $<100 \mathrm{~Hz}$. However, it should be noted that the published results on type I males (Weeg et al. 2002) were obtained using a shaker table system which primarily only delivers particle acceleration stimuli, whereas in our study we used a speaker, which delivers both particle motion and pressure stimuli. Further tests will need to be conducted using a shaker system to more accurately compare the particle acceleration sensitivities for the three midshipman sexual phenotypes.

\section{Potential mechanisms for the plasticity of saccular sensitivity}

The underlying mechanism(s) responsible for the seasonal reproductive-dependent changes in saccular sensitivity of type II males is likely similar to that proposed for females and type I males. Previous studies by Rohmann and Bass (2011) and Rohmann et al. (2013) showed that the seasonal variation in the frequency sensitivity of saccular hair cells in type I males could be explained by seasonal changes in the abundance of calcium-activated potassium BK channels, which are known to be responsible for a large conductance, outward current that produces electrical receptor oscillations along the hair cell epithelium (Lewis and Hudspeth 1983; Roberts et al. 1988; Fettiplace and Fuchs 1999). The electrical resonance that arises from the ion-channel current kinetics of hair cells is thought to be the major contributing factor that influences low-frequency $(<1 \mathrm{kHz})$ hair cell tuning in reptiles and birds (Fettiplace and Fuchs 1999) and in fish (Steinacker and Romero 1991, 1992). Rohmann et al. (2013) showed that by using the BK channel antagonist iberiotoxin to reduce BK channel availability they could effectively induce a saccular neurophysiological phenotype similar to that found in non-reproductive type I males. Thus, the BK channels of hair cells are thought to be a primary mechanism that can account for the seasonal changes in the frequency selectivity of hair cells in males 
and females. Another potential mechanism responsible for seasonal changes in saccular sensitivity is the reproductive state-dependent increases in saccular hair cell density and decreases in hair cell death observed in females by Coffin et al. (2012). Such seasonal increases in saccular hair cell density were independent of body size (age) and not observed in the other inner ear end organs, the lagena and utricle. In addition, Coffin et al. (2012) also observed in reproductive females that the increase in saccular hair cell density was concurrent with increases in overall magnitude of the evoked saccular potentials and decreases in auditory saccular thresholds (.i.e., increased auditory sensitivity). The above-mentioned mechanisms potentially responsible for the observed seasonal changes in saccular sensitivity have been proposed to be induced by estrogens and androgens acting via long term signaling pathways to upregulate BK channels and modulate hair cell proliferation and death to ultimately enhance the hearing sensitivity in this species (Coffin et al. 2012; Forlano et al. 2015, 2016). However, this steroid-mediated mechanism hypothesis remains to be tested.

In summary, our results indicate that the seasonal plasticity of auditory sensitivity previously reported for females and type I males also occurs in type II male midshipman. This form of reproductive state-dependent plasticity of auditory sensitivity provides an adaptive mechanism that enhances hearing sensitivity in type II males during the reproductive season. Such an increase in auditory sensitivity should increase the probability of conspecific detection by type II sneaker males and facilitate eavesdropping of type I male vocalizations for the assessment of type I male rivals and the selection of cuckoldry sites during the breeding season.

Acknowledgements The authors would like to thank the members of the Sisneros Lab and Captain Charlie Eaton and his crew of the $\mathrm{R} / \mathrm{V}$ Kittiwake for field assistance in animal collections. This work was supported by an NIH Auditory Neuroscience Training Grant 2T32DC005361-11 (to A. A. B. and E. A. W.) and a University of Washington Bridge Fund (to J. A. S.). All experimental procedures were approved by the University of Washington Institutional Animal Care and Use Committee and followed the National Institutes of Health guidelines for the care and use of animals.

\section{References}

Alderks PW, Sisneros JA (2011) Ontogeny of auditory saccular sensitivity in the plainfin midshipman fish, Porichthys notatus. J Comp Physiol A 197(4):387-398

Bass AH, Clark CW (2003) The physical acoustics of underwater sound communication. In: Simmons AM, Popper AN, Fay RR (eds) Acoustic communication. Springer, New York, pp 15-64

Bass AH, Ladich F (2008) Vocal-acoustic communication: from neurons to behavior. In: Webb JF, Popper AN, Fay RR (eds) Fish bioacoustics. Springer, New York, pp 253-278
Bass AH, McKibben JR (2003) Neural mechanisms and behaviors for acoustic communication in teleost fish. Progr Neurobiol 69(1): $1-26$.

Bass AH, Bodnar DA, Marchaterre MA (1999) Complementary explanations for existing phenotypes in an acoustic communication system. In: Hauser MD, Konishi M (eds) The design of animal communication. MIT, Cambridge, pp 493-514

Blaxter JHS (1981) The swim bladder and hearing. In: Tavolga WN, Popper AN, Fay RR (eds) Hearing and sound communication in fishes. Springer, Berlin, pp 61-72

Brantley RK, Bass AH (1994) Alternative male spawning tactics and acoustic signals in the plainfin midshipman fish Porichthys notatus Girard (Teleostei, Batrachoididae). Ethology 96(3):213-232

Braun CB, Grande T (2008) Evolution of peripheral mechanisms for the enhancement of sound reception. In: Webb JF, Popper AN, Fay RR (eds) Fish bioacoustics, Springer, New York, pp 99-144

Casper BM, Mann DA (2006) Evoked potential audiograms of the nurse shark (Ginglymostoma cirratum) and the yellow stingray (Urobatis jamaicensis). Environ Biol Fish 76(1):101-108

Chapman CJ, Sand O (1974) Field studies of hearing in two species of flatfish Pleuronectes platessa (L.) and Limanda limanda (L.) (Family Pleuronectidae). Comp Biochem Physiol A 47(1):371-385

Coffin AB, Mohr RA, Sisneros JA (2012) Saccular-specific hair cell addition correlates with reproductive state-dependent changes in the auditory saccular sensitivity of a vocal fish. J Neurosci 32(4):1366-1376

Cohen MJ, Winn HE (1967) Electrophysiological observations on hearing and sound production in the fish, Porichthys notatus. J Exp Zool 165(3):355-369

Coombs S, Popper AN (1979) Hearing differences among Hawaiian squirrelfish (family Holocentridae) related to differences in the peripheral auditory system. J Comp Physiol 132(3):203-207

De Vries HL (1950) The mechanics of the labyrinth otoliths. Acta Otolaryngol 38(3):262-273

Edds-Walton PL, Arruda J, Fay RR, Ketten DR (2015) Computerized tomography of the otic capsule and otoliths in the oyster toadfish, Opsanus tau. J Morphol 276(2):228-240

Fay RR (1984) The goldfish ear codes the axis of acoustic particle motion in three dimensions. Science 225(4665):951-954

Fay RR, Popper AN (1980) Structure and function in teleost auditory systems. In: Popper AN, Fay RR (eds) Comparative studies of hearing in vertebrates. Springer, Berlin, pp 3-42

Fay RR, Popper AN (2012) Fish hearing: new perspectives from two 'senior' bioacousticians. Brain Behav Evol 79(4):215-217

Fettiplace R, Fuchs PA (1999) Mechanisms of hair cell tuning. Annu Rev Physiol 61:809-834

Fine ML, Lenhardt ML (1983) Shallow-water propagation of the toadfish mating call. Comp Biochem Physiol A 76:225-231

Fine ML, Parmentier E (2015) Mechanisms of fish sound production. In: Ladich F (ed) Sound communication in fishes. Animal signals and communication, vol 4. Springer, Berlin, pp 77-126

Forlano PM, Sisneros JA, Rohmann KN, Bass AH (2015) Neuroendocrine control of seasonal plasticity in the auditory and vocal systems of fish. Front Neuroendocrinol 37:129-145.

Forlano PM, Maruska KP, Sisneros JA, Bass AH (2016) Hormonedependent plasticity of auditory systems in fishes. In: Bass AH, Sisneros JA, Popper AN, Fay RR (eds) Hearing and hormones. Springer International, New York, pp 15-51

Greenwood PH (1970) Skull and swimbladder connections in the fishes of the family Megalopidae. Bull British Mus Nat Hist (Zool) 14:121-135

Grober MS, Fox SH, Laughlin C, Bass AH (1994) GnRH cell size and number in a teleost fish with two male reproductive morphs: sexual maturation, final sexual status and body size allometry. Brain Behav Evol 43(2):61-78 
Hawkins AD (1993) Underwater sound and fish behavior. In: Pitcher TJ (ed) The behaviour of teleost fishes. Chapman and Hall, London, pp 114-151

Horodysky AZ, Brill RW, Fine ML, Musick JA, Latour RJ (2008) Acoustic pressure and particle motion thresholds in six sciaenid fishes. J Exp Biol 211(9):1504-1511

Jerko H, Turunen-Rise I, Enger PS, Sand O (1989) Hearing in the eel (Anguilla anguilla). J Comp Phys A 165(4):455-459

Kelley DB, Bass AH (2010) Neurobiology of vocal communication: mechanisms for sensorimotor integration and vocal patterning. Curr Opin Neurobiol 20(6):748-753

Ladich F (2004) Sound production and acoustic communication. In: von der Emde G, Mogdans J, Kapoor BG (eds) The senses of fishes: adaptations for the reception of natural stimuli. Narosa Publishing House Pvt Ltd, New Delhi, pp 210-230

Lewis RS, Hudspeth AJ (1983) Voltage-and ion-dependent conductances in solitary vertebrate hair cells. Nature 304:538-541

Maruska KP, Sisneros JA (2015) Sex steroid-dependent modulation of acoustic communication systems in fishes. In: Ladich F (ed) Sound communication in fishes. Springer, New York, pp 207-233

McKibben JR, Bass AH (1998) Behavioral assessment of acoustic parameters relevant to signal recognition and preference in a vocal fish. J Acoust Soc Am 104(6):3520-3533

McKibben JR, Bass AH (1999) Peripheral encoding of behaviorally relevant acoustic signals in a vocal fish: single tones. J Comp Physiol A 184(6):563-576

McKibben JR, Bass AH (2001) Peripheral encoding of behaviorally relevant acoustic signals in a vocal fish: harmonic and beat stimuli. J Comp Physiol A 187(4):271-285

Popper AN, Fay RR (1993) Sound detection and processing by fish: critical review and major research questions. Brain Beh Evol 41:14-25

Popper AN, Fay RR (2011) Rethinking sound detection by fishes. Hear Res 273(1):25-36

Roberts WM, Howard J, Hudspeth AJ (1988) Hair cells: transduction, tuning, and transmission in the inner ear. Ann Rev Cell Biol 4(1):63-92

Rogers PH, Cox M (1988) Underwater sound as a biological stimulus. In: Atema J, Fay RR, Popper AN, Tavolga WN, Sensory biology of aquatic animals. Springer, New York, pp 131-149

Rohmann KN, Bass AH (2011) Seasonal plasticity of auditory hair cell frequency sensitivity correlates with plasma steroid levels in vocal fish. J Exp Biol 214(11):1931-1942

Rohmann KN, Fergus DJ, Bass AH (2013) Plasticity in ion channel expression underlies variation in hearing during reproductive cycles. Curr Biol 23(8):678-683

Schellart NAM, Popper AN (1992) Functional aspects of the evolution of the auditory system of actinopterygian fish. In: Webster
DB, Fay RR, Popper AN (eds) The evolutionary biology of hearing. Springer, New York, pp 295-322

Sisneros JA (2007) Saccular potentials of the vocal plainfin midshipman fish, Porichthys notatus. J Comp Physiol A 193:413-424

Sisneros JA (2009a) Adaptive hearing in the vocal plainfin midshipman fish: getting in tune for the breeding season and implications for acoustic communication. Integr Zool 4:33-42

Sisneros JA (2009b) Seasonal plasticity of auditory saccular sensitivity in the vocal plainfin midshipman fish, Porichthys notatus. J Neurophysiol 102(2):1121-1131

Sisneros JA, Bass AH (2003) Seasonal plasticity of peripheral auditory frequency sensitivity. J Neurosci 23:1049-1058

Sisneros JA, Bass AH (2005) Ontogenetic changes in the response properties of individual, primary auditory afferents in the vocal plainfin midshipman fish Porichthys notatus Girard. J Exp Biol 208(16):3121-3131

Sisneros JA, Rogers PH (2016) Directional Hearing and Sound Source Localization in Fishes. In: Sisneros JA (ed) Fish hearing and bioacoustics. Springer International, New York, pp 121-155

Sisneros JA, Forlano PM, Knapp R, Bass AH (2004) Seasonal variation of steroid hormone levels in an intertidal-nesting fish, the vocal plainfin midshipman. Gen Comp Endocrinol 136:101-116

Steinacker A, Romero A (1991) Characterization of voltage-gated and calcium-activated potassium currents in toadfish saccular hair cells. Brain Res 556(1):22-32

Steinacker A, Romero A (1992) Voltage-gated potassium current and resonance in the toadfish saccular hair cell. Brain Res 574:229-236

Tomkins JL, Simmons LW (2002). Measuring relative investment: a case study of testes investment in species with alternative male reproductive tactics. Anim Behav 63(5):1009-1016

Tricas TC, Boyle KS (2015) Sound pressure enhances the hearing sensitivity of Chaetodon butterfly fishes on noisy coral reefs. J Exp Biol 218:1585-1595

Vasconcelos RO, Sisneros JA, Amorim MCP, Fonseca PJ (2011) Auditory saccular sensitivity of the vocal Lusitanian toadfish: low frequency tuning allows acoustic communication throughout the year. J Comp Physiol A 197(9):903-913

Weeg M, Fay RR, Bass AH (2002) Directionality and frequency tuning of primary saccular afferents of a vocal fish, the plainfin midshipman (Porichthys notatus). J Comp Physiol A 188(8):631-641

Whitchurch EA, Ketten DR, Forlano PM, Fay RR, Sisneros JA (2012) Swim bladder sexual dimorphism in the plainfin midshipman fish: Implications for acoustic communication in this species. Program No. 296.11/CCC73. 2012 Neuroscience Meeting Planner. Society for Neuroscience, New Orleans (online)

Wysocki LE, Codarin A, Ladich F, Picciulin M (2009) Sound pressure and particle acceleration audiograms in three marine fish species from the Adriatic Sea. J Acoust Soc Am 126(4):2100-2107 